\title{
Red wine flavonoids and vascular health
}

\author{
Jonathan M. Hodgson* \\ School of Medicine and Pharmacology, University of Western Australia, Western Australia, Australia
}

\begin{abstract}
Relative to non-drinkers, a low to moderate alcohol intake is associated with lower risk of cardiovascular and total mortality. Epidemiological studies also suggest that a low to moderate red wine consumption may protect against cardiovascular disease. Because epidemiological studies show a similar relationship for different types of alcoholic beverages, the alcohol (ethanol), at low to moderate intakes, is proposed to provide protection. However, it remains uncertain whether there is a causal link between alcohol generally or red wine specifically and cardiovascular disease. This is very difficult to explore in epidemiological studies because of the many confounding social and dietary factors, and because risk of cardiovascular disease increases substantially from moderate to very high intakes of alcohol.

The other major components of red wine that have received attention as potentially cardioprotective are the flavonoids. There is mounting evidence that flavonoids and foods and beverages rich in flavonoids can make an important contribution to cardiovascular health. Fruit and vegetables, tea and cocoa are important sources of flavonoids in the human diet. The intake of these foods hasl been associated with reduced risk of cardiovascular disease in population studies.

Flavonoids are potent antioxidants in vitro, but it is their ability to cause vasorelaxation that is likely to be important for any vascular health benefits. Red grapes, their skin, their seeds and the wine derived from them are rich in flavonoids. Population studies have found that higher intakes of flavonoids are associated with lower risk for cardiovascular disease. In vitro studies, studies using animal models and human intervention studies have investigated how flavonoids might contribute to reduced risk of cardiovascular disease. A variety of mechanisms and outcomes have been explored and there is now strong evidence that flavonoids can improve endothelial function. A number of human studies have been performed to investigate the in vivo effects of red wine derived flavonoids on endothelial function. The results of these studies are mixed, with several studies indicating acute improvements, while other studies suggest little benefit of regular short-term consumption of red wine flavonoids. The effects of other rich dietary sources of flavonoids, such as tea and cocoa, to improve endothelial function may also provide a guide to the potential benefits of red wine flavonoids. Results of human trials and meta-analyses of these trials suggest that tea, cocoa and flavonoid-rich fruits can improve endothelial function.

Therefore there is good evidence that flavonoid-rich foods and beverages can have vascular health benefits. However, because bioactivity of different flavonoids varies, health effects cannot be generalized to all flavonoids and flavonoid-rich foods. Further studies are needed to establish any vascular health benefits of the red wine flavonoids.
\end{abstract}

Keywords: Red wine, flavonoids, cardiovascular disease, endothelial function, vascular health

\section{Background: The epidemiology}

Interest in the effects of alcohol on human health has remained strong for more than a century. Many epidemiological studies have now evaluated the relationship between alcohol intake and health outcomes. It is clear that heavy alcohol intake substantially

*Corresponding author: Dr. Jonathan M. Hodgson, School of Medicine and Pharmacology, GPO Box X2213 Perth, Western Australia 6847, Australia. Tel.: +61 6189224 0267; Fax: +61 6189224 0246; E-mail: Jonathan.Hodgson@uwa.edu.au. increases the risk of a range of chronic diseases [1, 2]. However, there is now considerable support for the idea that low to moderate alcohol intake is associated with lower risk of cardiovascular and total mortality. Relative to non-drinkers, low to moderate alcohol consumption is associated with a 20 to $30 \%$ lower risk of cardiovascular mortality and a 10 to $15 \%$ lower risk of all-cause mortality [2].

It remains uncertain whether this link is causal. This is very difficult to explore in epidemiological studies because of the many confounding social and dietary factors, and because risk of cardiovascular disease 
increases substantially from moderate to very high intakes of alcohol. However, there is evidence that components of alcohol, including the ethanol itself and polyphenols can influence risk factors and processes linked to vascular disease [1]. These include potential effects on endothelial function, blood pressure, inflammation, platelet function and thrombosis, oxidative stress, glucose metabolism and the lipid profile.

There has also been interest in possible differences in effects of different types of alcoholic beverage (wine, beer, spirits). Studies that have compared the different beverages indicate that wine, and red wine particularly is often more strongly related to benefit than other forms of alcohol $[1,3]$. This may be due to confounding factors related to red wine consumption. It is also possible that red wine may contain higher levels of protective components. The polyphenols, including flavonoids, are important components of red wine that have received attention as potentially cardioprotective.

\section{Red wine flavonoids}

The flavonoids present in red wine include a wide range of compounds that affect colour, taste and mouth feel of the wine. Flavonoids are the main polyphenols present in red wine by weight, and constitute about 80 to $90 \%$ of total polyphenols. Flavonoids are derived primarily from the skin and or seed of red grapes. Other polyphenols also present in red wine include the phenolic acids (caffeic and cinnamic acids) and stilbenoids (resveratrol).

The major flavonoids in red wine include flavonols, flavan-3-ol monomers (catechins), flavan-3-ol polymers (proanthocyanidins) and anthocyanidins. One of the main flavonols is quercetin, which can be absorbed intact and metabolized to a variety of metabolites [4]. The skin of red grapes are rich in flavan-3-ol monomers such as catechin, epicatechin, gallocatechin and epigallocatechin, whereas the seeds contain mainly proanthocyanidins [5]. Flavan-3-ol monomers can be absorbed without modification, but the proanthocyanidins are poorly absorbed. They are largely metabolized to phenolic acids, which may then be absorbed. The anthocyanidins, which may also be absorbed intact [6] contribute to skin colour, and increase in concentration as the grape ripens. Differences in structure, absorption and metabolism of the different flavonoids could influence bioactivity.

\section{Flavonoid intake and cardiovascular disease}

There is increasing evidence that a higher flavonoid intake is associated with a lower risk of cardiovascular disease in populations. A number of population studies have investigated the relationships of specific classes of flavonoids with cardiovascular disease risk. High flavonols (quercetin) intake is consistently associated with lower risk [7]. Recent studies have investigated relationships of several flavonoid classes and indicate that flavan-3-ols and anthocyanidins may also benefit cardiovascular disease outcomes $[8,9]$. These studies indicate that several different classes of flavonoids could contribute to lower risk of cardiovascular disease.

\section{Bioactivity of flavonoids}

In vitro studies, studies using animal models and human intervention studies have investigated how flavonoids might contribute to reduced risk of cardiovascular disease. A variety of mechanisms and outcomes have been explored, including the effects on endothelial function, blood pressure, oxidative stress, inflammation, cholesterol reduction, homocysteine metabolism, platelet function, body fatness and type 2 diabetes [10]. For most of the outcomes there is inconsistent and or limited data. However, there is now a substantial body of evidence that flavonoids can enhance nitric oxide (a molecule that causes vascular relaxation) status and improve endothelial function [10].

\section{Endothelial function and its measurement}

The endothelium is the inner lining of blood vessels. It functions as a selectively permeable barrier between blood and tissues. Normal endothelial function has a number of roles and includes regulation of vasomotor tone primarily through the release of nitric oxide. Nitric oxide is one of the most important molecules released by the endothelium. Endothelial dysfunction is characterized by the loss of normal endotheliumdependent and NO-mediated vasodilation in the artery. Endothelial dysfunction is likely to contribute to the pathogenesis of cardiovascular disease, and it is considered as an early biomarker for the development of cardiovascular disease [11]. 
Endothelial function may be assessed in a number of ways. Isolated vessels from animals can be used to assess the effects of potentially vasoactive substances in vitro. In humans, one of the main methods has been to use ultrasonography to measure flow-mediated dilatation of conduit vessels, such as the brachial artery. This is a non-invasive technique that measures NOdependent vasodilation of the artery in response to shear stress induced by increased blood flow [12].

\section{Red wine flavonoids: Effects on endothelial function}

A number of studies have investigated effects of alcohol or red wine containing alcohol on endothelial function [13, 14]. Although alcohol raises blood pressure [15], there is little evidence that red wine containing alcohol impairs endothelial function $[16,17]$. In order to establish if red wine flavonoids influence endothelial function, effects must be assessed in the absence of alcohol using dealcoholized red wine, red grape juice, or grape seed or skin extracts. In studies using animal models, red grape derived flavonoids have been shown to improve endothelial function [18]. A number of human studies have also been performed to investigate the in vivo effects of short-term supplementation with dealcoholized red wine, red grape juice, or grape seed or skin extracts on endothelial function [14]. Most of these studies have assessed endothelial function by measuring flow-mediated dilatation of the brachial artery. This is mediated primarily by nitric oxide. A low flow-mediated dilatation is associated with higher risk of cardiovascular disease events [19].

Studies exploring the effects of red wine flavonoids on endothelial function can be categorized according to whether effects were investigated in an acute (including postprandial) setting or following shortterm regular consumption. In the acute setting most studies have found that dealcoholized red wine or flavonoid-rich red grape/wine extracts result in significant improvement in endothelial function [14]. In short-term trials, early studies found that purple grape juice $[20,21]$ or grape seed extract [22] resulted in improved endothelial function. However, a number of more recent trials have found no effect of shortterm regular consumption of red wine flavonoids [16, 17, 23-25]. Therefore, the results of these studies are mixed, with some studies indicating benefit and others showing no significant effect. Additional studies are needed in order to establish the effects of regular consumption of a diet higher in red wine flavonoids on endothelial function.

\section{Flavonoids from other sources: Effects on endothelial function}

In order to further evaluate the potential of red wine derived flavonoids on endothelial function it is useful to explore the effects of other foods and beverages that contain the same of similar flavonoids to those present in red wine. In the human diet flavonols, flavan-3-ols and anthocyanidins can be derived from tea, fruits and chocolate (cocoa).

\subsection{Tea}

The term 'tea' refers to the plant Camellia sinensis, its leaves, and infusions derived from them. The two main types of tea are green and black. Both green and black teas are rich dietary sources of flavonoids, and contain both flavonols such as quercetin and monomeric flavan-3-ols (mainly the catechins). Tea intake has been associated with reduced risk of cardiovascular disease in cross-sectional and prospective population studies [10]. These studies have led on to investigations of the effects of tea on endothelial function.

There is now consistent data showing that tea and tea flavonoids can enhance nitric oxide status [26] and improve endothelial function [10]. Most studies have used black tea, and the effects have been investigated both acutely and following short-term regular ingestion. A recent meta-analysis of these studies found that tea consumption resulted in an overall improvement in flow-mediated dilatation of the brachial artery by approximately $30 \%$ [27]. Benefits have been observed in both acute and short-term studies. There is also some evidence for a dose-response relationship. It is suggested that these effects may be at least partly responsible for benefits of tea and tea flavonoids on cardiovascular health [10].

\subsection{Cocoa/chocolate}

Cocoa is one of the richest sources of flavonoids. It is rich in the monomeric flavan-3-ol epicatechin, but also contains significant levels of proanthocyanidins. Cocoa is manufactured to cocoa powder and chocolate. 
Although some of the flavonoids are lost during this process, some chocolate can still provide flavonoids to the diet. Early interest in cocoa derived from observations in Kuna Indians, who consume considerable amounts of cocoa daily as a beverage, and are protected against an age-dependent increase in blood pressure. This is often against the background of a high salt diet. Recent population studies have investigated effects of cocoa/chocolate intake on cardiovascular outcomes in populations. These studies suggest that modest regular cocoa/chocolate intake is associated with a 10 to $50 \%$ lower risk of cardiovascular outcomes [28]. Observations in the Kuna Indians have also led to research on the effects of cocoa flavonoids on endothelial function.

About ten studies have now assessed the effect of flavonoid-rich cocoa or dark chocolate on endothelial function assessed using flow-mediated dilatation of the brachial artery. A meta-analyses of these studies found that flavonoid-rich cocoa or dark chocolate can improve endothelial function assessed using flowmediated dilatation by approximately $\sim 40 \%$ [29]. The magnitude of these effects is similar to those observed for tea and those observed in acute studies with red wine flavonoids.

\section{Flavonoids and endothelial function: A meta-analysis}

The evidence that flavonoids derived from some sources such as tea and cocoa can enhance endothelial function is now quite strong. The evidence for benefit of red wine derived flavonoids is less consistent. However, red wine contains many of the same compounds as those present in tea, cocoa and fruits shown to improve endothelial function. These include the flavonols, flavan-3-ols (monomers and polymers) and anthocyanidins. A recent systematic review and meta-analysis of randomised controlled trials of flavonoid-rich food products has attempted to explore the effects of different flavonoid classes on vascular function [30]. All (combined) flavonoid subclasses resulted in a significant improvement in flow-mediated dilatation by approximately $30 \%$ in acute studies and approximately $10 \%$ in short-term studies of regular ingestion of the flavonoids [30]. These effects would be consistent with the suggested benefit of red wine flavonoids in acute studies, with less support for benefit in studies of regular ingestion $[14,30]$. Kay et al [30] also explored estimated effects of individual flavonoid subclasses. They found that the estimated impact of the classes of flavonoids present in red wine (flavonols, flavan-3-ols and anthocyanidins) was similar or larger than that for total flavonoids. This was for both acute and short-term studies.

\section{Summary}

In population studies low to moderate red wine consumption is associated with protection against cardiovascular disease. It is suggested that an important protective component of red wine are the flavonoids. There is mounting evidence that flavonoids and foods rich in flavonoids can make a contribution to cardiovascular health. Intakes of fruit and vegetables, tea and cocoa have all been associated with reduced risk of cardiovascular disease in population studies. Furthermore, population studies have also linked the intake of specific flavonoids to lower risk of cardiovascular disease. A variety of mechanisms related to benefit on cardiovascular health have been explored. For most of the outcomes there is inconsistent and or limited data. However, there is now a substantial body of evidence that flavonoids can improve endothelial function.

Red grapes, their skin, their seeds and the wine derived from them are rich in flavonoids. Flavonoids may have a number of bioactivities including potent antioxidant activity in vitro. However, it is their ability to cause vasorelaxation that is likely to be important for any vascular health benefits. Human studies that have investigated the acute and short-term effects of red wine flavonoid (without alcohol) are inconsistent. They do suggest the potential for acute improvements in flow-mediated dilatation of the brachial artery, but the evidence for benefit with regular ingestion remains weak. The demonstrated benefits of other rich dietary sources of flavonoids, such as tea, cocoa and other fruits on endothelial function also provide a guide to the potential benefits of red wine flavonoids. This is primarily because many of the flavonoids present in red wine are also present in these other foods. A recent meta-analysis that pooled results of trials of flavonoidrich foods suggests that the major flavonoids present in red wine could improve endothelial function.

\section{Conclusion}

There is good evidence that flavonoid-rich foods and beverages can have vascular health benefits. Because 
bioactivity of different flavonoids varies, health effects cannot be generalized to all flavonoids and flavonoidrich foods. However, there is supporting evidence from studies using a range of food and beverage sources of flavonoids that the flavonoids present in red wine could contribute to vascular health. Further studies are needed to establish any vascular health benefits of the regular consumption of red wine flavonoids.

\section{References}

[1] Arranz S, Chiva-Blanch G, Valderas-Martinez P, MedinaRemon A, Lamuela-Raventos RM, Estruch R. Wine, beer, alcohol and polyphenols on cardiovascular disease and cancer. Nutrients. 2012;4(7):759-81.

[2] Ronksley PE, Brien SE, Turner BJ, Mukamal KJ, Ghali WA. Association of alcohol consumption with selected cardiovascular disease outcomes: A systematic review and meta-analysis. Bmj. 2011;342:d671.

[3] Di Castelnuovo A, Rotondo S, Iacoviello L, Donati MB, De Gaetano G. Meta-analysis of wine and beer consumption in relation to vascular risk. Circulation. 2002;105(24):2836-44.

[4] Terao J, Murota K, Kawai Y. Conjugated quercetin glucuronides as bioactive metabolites and precursors of aglycone in vivo. Food \& Function. 2011;2(1):11-7.

[5] Mattivi F, Vrhovsek U, Masuero D, Trainotti D. Differences in the amount and structure of extractable skin and seed tannins amongst red grape cultivars. Australian Journal of Grape and Wine Research. 2009;15:27-35.

[6] Mazza G, Kay CD, Cottrell T, Holub BJ. Absorption of anthocyanins from blueberries and serum antioxidant status in human subjects. Journal of Agricultural \& Food Chemistry. 2002;50(26):7731-7.

[7] Huxley RR, Neil HA. The relation between dietary flavonol intake and coronary heart disease mortality: A meta-analysis of prospective cohort studies. Eur J Clin Nutr. 2003;57(8):904-8.

[8] Mink PJ, Scrafford CG, Barraj LM, Harnack L, Hong CP, Nettleton JA, et al. Flavonoid intake and cardiovascular disease mortality: A prospective study in postmenopausal women. American Journal of Clinical Nutrition. 2007;85(3):895-909.

[9] Mursu J, Nurmi T, Tuomainen TP, Salonen JT, Pukkala E, Voutilainen S. Intake of flavonoids and risk of cancer in Finnish men: The Kuopio Ischaemic Heart Disease Risk Factor Study. Int J Cancer. 2008;123(3):660-3.

[10] Hodgson JM, Croft KD. Tea flavonoids and cardiovascular health. Mol Aspects Med. 2010;31(6):495-502.

[11] Schroeder S, Enderle MD, Ossen R, Meisner C, Baumbach A, Pfohl M, et al. Noninvasive determination of endotheliummediated vasodilation as a screening test for coronary artery disease: Pilot study to assess the predictive value in comparison with angina pectoris, exercise electrocardiography, and myocardial perfusion imaging. Am Heart J. 1999;138(4 Pt 1):731-9.

[12] Woodman RJ, Watts GF. Measurement and application of arterial stiffness in clinical research: Focus on new methodologies and diabetes mellitus. Med Sci Monit. 2003;9(5):RA81-9.
[13] Puddey IB, Zilkens RR, Croft KD, Beilin LJ. Alcohol and endothelial function: A brief review. Clin Exp Pharmacol Physiol. 2001;28(12):1020-4.

[14] Karatzi K, Karatzis E, Papamichael C, Lekakis J, Zampelas A. Effects of red wine on endothelial function: Postprandial studies vs clinical trials. Nutr Metab Cardiovasc Dis. 2009;19(10):744-50.

[15] Chen L, Davey Smith G, Harbord RM, Lewis SJ. Alcohol intake and blood pressure: A systematic review implementing a Mendelian randomization approach. PLoS Med. 2008;5(3):e52.

[16] Zilkens RR, Burke V, Hodgson JM, Barden A, Beilin LJ, Puddey IB. Red wine and beer elevate blood pressure in normotensive men. Hypertension. 2005;45(5):874-9.

[17] Zilkens RR, Rich L, Burke V, Beilin LJ, Watts GF, Puddey IB. Effects of alcohol intake on endothelial function in men: A randomized controlled trial. J Hypertens. 2003;21(1):97-103.

[18] Chan SL, Capdeville-Atkinson C, Atkinson J. Red wine polyphenols improve endothelium-dependent dilation in rat cerebral arterioles. J Cardiovasc Pharmacol. 2008;51(6):553-8.

[19] Inaba Y, Chen JA, Bergmann SR. Prediction of future cardiovascular outcomes by flow-mediated vasodilatation of brachial artery: A meta-analysis. Int J Cardiovasc Imaging. 2010;26(6):631-40.

[20] Chou EJ, Keevil JG, Aeschlimann S, Wiebe DA, Folts JD, Stein JH. Effect of ingestion of purple grape juice on endothelial function in patients with coronary heart disease. Am J Cardiol. 2001;88(5):553-5.

[21] Stein JH, Keevil JG, Wiebe DA, Aeschlimann S, Folts JD. Purple grape juice improves endothelial function and reduces the susceptibility of LDL cholesterol to oxidation in patients with coronary artery disease. Circulation. 1999;100(10): 1050-5.

[22] Clifton PJ. Effect of grape seed extract and quercetin on cardiovascular and endothelial parameters in high-risk subjects. Journal of Biomedicine and Biotechnology. 2004;5:272-8.

[23] Ward NC, Hodgson JM, Croft KD, Burke V, Beilin LJ, Puddey IB. The combination of vitamin $\mathrm{C}$ and grape-seed polyphenols increases blood pressure: A randomized, double-blind, placebo-controlled trial. J Hypertens. 2005;23(2):427-34.

[24] van Mierlo LA, Zock PL, van der Knaap HC, Draijer R. Grape polyphenols do not affect vascular function in healthy men. J Nutr. 2010;140(10):1769-73.

[25] Mellen PB, Daniel KR, Brosnihan KB, Hansen KJ, Herrington DM. Effect of muscadine grape seed supplementation on vascular function in subjects with or at risk for cardiovascular disease: A randomized crossover trial. J Am Coll Nutr. 2010;29(5):469-75.

[26] Loke WM, Hodgson JM, Proudfoot JM, McKinley AJ, Puddey IB, Croft KD. Pure dietary flavonoids quercetin and (-)-epicatechin augment nitric oxide products and reduce endothelin-1 acutely in healthy men. American Journal of Clinical Nutrition. 2008;88(4):1018-25.

[27] Ras RT, Zock PL, Draijer R. Tea consumption enhances endothelial-dependent vasodilation; a meta-analysis. PLoS ONE. 2011;6(3):e16974.

[28] Buitrago-Lopez A, Sanderson J, Johnson L, Warnakula S, Wood A, Di Angelantonio E, et al. Chocolate consumption and cardiometabolic disorders: Systematic review and metaanalysis. Bmj. 2011;343:d4488. 
[29] Hooper L, Kay C, Abdelhamid A, Kroon PA, Cohn JS, Rimm EB, et al. Effects of chocolate, cocoa, and flavan-3-ols on cardiovascular health: A systematic review and meta-analysis of randomized trials. American Journal of Clinical Nutrition. 2012;95(3):740-51.
[30] Kay CD, Hooper L, Kroon PA, Rimm EB, Cassidy A. Relative impact of flavonoid composition, dose and structure on vascular function: A systematic review of randomised controlled trials of flavonoid-rich food products. Mol Nutr Food Res. 2012;56(11):1605-16. 